\title{
Similarity index: A procedure for comparing impact time histories validated with soft impact test
}

\author{
J. Alonso*, J.A. Parra, A. Pacios, M.C. Huerta \\ Departamento de Ingeniería Mecánica, Universidad Politécnica de Madrid, C/ José Gutiérrez Abascal, 2, 28006 Madrid, Spain
}

A R T I C L E I N F O

\section{Keywords:}

Impact force

Similarity index

Glass testing

2DOF

\begin{abstract}
A B S T R A C T
This paper describes the procedure developed to compare the dynamic behavior of a structure due to an impact load. The comparison procedure is especially useful for controlling the impact level and repeatability during testing, but it can also be used for fine-tuning the process of simplified models or finite element models. Both features provide important engineering value and permit the assessment of transient impact histories, qualitatively and quantitatively. More than 240 soft impacts, from a human impact against glass test campaign, have been used to develop and validate the comparison method.

Three indicators have been defined in order to control the most significant impact parameters: the impact level (impulse), duration of the impact (stiffness), and maximum acceleration (peak system response). Based on the knowledge of the impact phenomena, the procedure defines a Similarity Index (SIn) that entails the weighted addition of the indicators. The SIn procedure assigns a numerical value to the difference between two time histories over the duration of the contact, and it is used to compare a series of data from a database of soft human impacts tests versus standard glass curves and curves obtained from a simplified 2DOF model.
\end{abstract}

\section{Introduction}

In recent years, many impact studies have been carried out to study the behavior of different structures through the analysis of transient responses from brittle materials such as glass [1] to more complex composite structures [2]. In addition, there are numerous studies that define simplified models for predicting the time histories of the contact stage for both soft [3] and hard impacts [4]. In the case of structural glass used in facades, floors, staircases or bridges [5], there are standards, as the German normative TRAV [6], that propose alternative studies (test or mathematical models) to verify the structural feasibility of a component.

In addition, there are many transient impact histories provided by finite element simulations [7]. Full transient simulations in finite element software are an alternative method for studying impact loads [8-10]. For example, in the definition of actual boundary conditions, engineers easily generate different transient results by modifying the model parameters and comparing the results to the reference $[11,12]$.

The existence of all these different sources of impacts creates the necessity for a procedure or a type of "tool" that allows comparing different time histories. The possibility of performing a qualitative and quantitative evaluation of the similarity between two registers, regardless of their origin, is helpful for design and research purposes. Another application is the comparison between test impacts with equal energy levels and the same structure in order to analyze the degree of repeatability.

Focusing on the human impact on building glass, there are some standards [13] that describe the time evolution of the impact head acceleration for some glass. Several time histories are presented in different studies that compare mathematical models [14] to finite element models [15] and simplified models [16]. To fine-tune these mathematical approaches, a quantified procedure for comparing these histories may be useful.

For comparison purpose, there are several mathematical techniques for error measurement that have been used for many years in the analysis of transient signals [17]. These techniques use magnitude and a phase error factor as parameters to evaluate the differences between two general functions. These procedures focus more on general signal analysis than the transient impact phenomenon. There are also examples of impact comparison in vehicle accidents [18], in which acceleration and velocity are compared to test data and finite element simulations. This study includes amplitude, phase, time shift and shape as parameters of similarity.

The aforementioned techniques are based on mathematical

\footnotetext{
* Corresponding author.

E-mail addresses: j.alonso@alumnos.upm.es (J. Alonso), jose.parra.hidalgo@alumnos.upm.es (J.A. Parra), antonia.pacios@upm.es (A. Pacios),
} 
comparisons of functions, but they do not allow evaluating the differences of the physical behavior of the phenomenon. Therefore, a method based on indicators of the physical behavior seems a better alternative for engineering activities.

This comparison of time histories may be applied to the dynamic phenomena that are currently used for dimensioning: hard impacts, soft impacts and explosions. To define an impact level, all types of impact tests [19] use the initial potential energy (height). This value is typically accompanied by its characteristic result values, duration and maximum values of the applied forces [3]. However, there are cases, especially in blast loading studies [20,21], in which the impulse of the applied force is one of the main parameters for the impact definition. This parameter was incorporated as a characteristic value of impact on glass in [22], which the adequacy of simplified a pproaches for predicting the response of laminated glass under blast load is discussed.

Current comparison techniques based on time histories are complex and do not focus on structural behavior. For example, in a soft impact test for glass [23], the use of different impact elements may change the time history response of the impact head during the contact stage with little influence on the structure. Therefore, a simple procedure focused on the structural behavior is useful in all types of comparisons of test impact and it could also be applied to the comparison of mathematical models.

This paper takes into consideration three parameters obtained from the acceleration of the impact element: duration (DP), maximum value (AP) and impulse (IP) of the applied force.

The objective of this paper is to present a method, using the DP, IP and AP parameters, to compare two impacts while deeming one as a reference (ImR) and the other as a study impact (ImS). To evaluate the impact histories, a similarity index has been defined based on the indicator quality of each parameter (IPI, IPA and IPD).

For this purpose, the human impact on building glass has been studied, using an exhaustive test campaign [24] including the criteria of standard UNE-12600 [25] to have enough information about the phenomenon.

To verify the applicability of the comparison method (index of similarity - indicators), data provided by standards, mathematical models and tests have been used. Two main studies are presented: repeatability comparison, using the records of the same impact obtained in the laboratory, and a general comparison between impacts with different conditions (impact level, boundary conditions of the structure and sizes).

\section{Description of phenomenon}

\subsection{Mathematical model}

Soft impact is a complex transient phenomenon in which one object strikes another with an initial energy. Throughout the process, and especially in the contact stage, there are many effects that can change the expected behavior of an impact element and a structure. Initial energy may not be applied to a tested structure due to measurement errors in the height, dissipation losses and energy distribution among the different components of the structure, impact element and support structures.

The duration of contact, one of the principal parameters of the phenomenon, is governed mainly by the stiffness of both a structure and an impact element. In many cases, non-linear effects might appear due to the stiffness variation as a function of the impact force (large displacement and non-linearities of the material).

During the contact stage, the force, transmitted by an impact element, increases until reaching its maximum, approximately in the middle of its duration. Once the maximum force is reached, it descends until the contact stage finishes. The i mpacted element $m$ aintains vibrating energy, while part of the energy is returned to the impact element that rebounds to a final height.

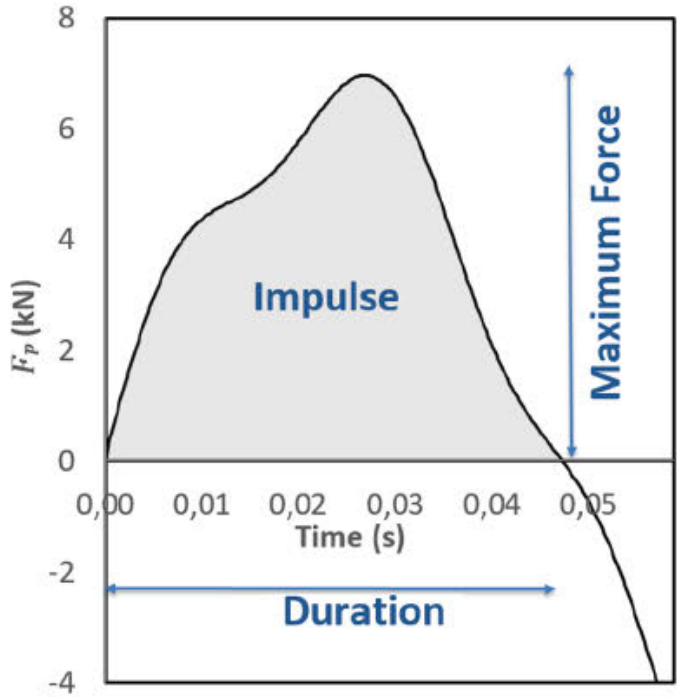

Fig. 1. Impact parameter description based on a 2DOF solution of a $10 \mathrm{~mm}$ tempered glass-all sides supported.

In tests, such as the glass impact test, some energy dissipates [25], and the amount of impulse is necessary to describe the impact [26]. Maximum force and duration are the other parameters required in order to complete the information on each impact. An example of the phenomena during the contact is presented on Fig. 1. The example is based on a 2DOF solution of an impact against a tempered glass of $10 \mathrm{~mm}$ of thickness with all sides supported.

To determine what occurs during an impact, the dynamic behavior of both elements (structure and impact element) is essential. Based on dynamic theory [27], the soft impact phenomenon may be reproduced with a 2DOF model [28]. For human impacts on glass plates, a 2DOF model (Fig. 2) is proposed, with one degree of freedom for the glass and another for the pendulum, defined by its mass $\left(m_{p}\right)$ and stiffness $\left(k_{p}\right)$.

The glass plate is defined by $k_{\mathrm{g}}(\mathrm{N} / \mathrm{m})$, the modal point stiffness of the first mode of vibration at the center of the glass plate (impact point) (Eq. (1)), and $m_{\mathrm{g}}(\mathrm{kg})$, the modal mass of the glass (Eq. (2)), where $\omega_{n g}$ $(\mathrm{rad} / \mathrm{s})$ is the natural frequency and $\phi_{\mathrm{cg}}$ is the modal component of the mid-point of the plate, normalized to $\varnothing_{c}{ }^{T} M \varnothing_{c}=I(\mathrm{~kg})$.

$k_{\mathrm{g}}=\frac{\omega_{n g}^{2}}{\left(\varnothing_{\mathrm{cg}} \cdot \varnothing_{\mathrm{cg}}\right)}$

$m_{\mathrm{g}}=\frac{k_{\mathrm{g}}}{\omega_{n g}^{2}}$

Natural frequency and normalized mode shape depend on the boundary conditions of the plate, as can be observed in test [29]. In order to use more accurate results for the specimens used in this work, these values are obtained by a modal test.

Glass plate behavior can be non-linear due to its large displacement. An equivalent linear stiffness, depending on the impact level, may be defined to calculate an impact solution. In reference [30], there is a description of the hypotheses used to modify the stiffness of the 2DOF

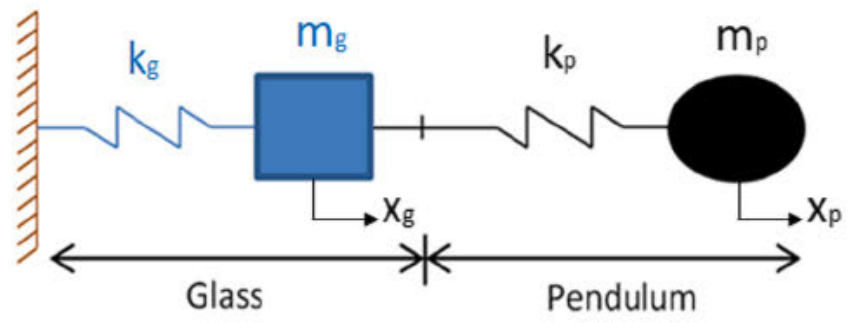

Fig. 2. 2DOF simplified model. 
simplified model in order to represent the non-linear effect produced by large displacement. Therefore, for each impact, a linear solution is performed with the corresponding linear equivalent stiffness.

The impact transient response can be solved (Eq. ((3)), during the contact phase, by a modal superposition of a 2DOF, thereby considering the initial velocity of the impact element as input during the contact phase.

$\left[\begin{array}{cc}m_{g} & 0 \\ 0 & m_{p}\end{array}\right] \cdot\left[\begin{array}{c}\ddot{x}_{g} \\ \ddot{x}_{p}\end{array}\right]+\left[\begin{array}{cc}k_{g}+k_{p} & -k_{p} \\ -k_{p} & k_{p}\end{array}\right] \cdot\left[\begin{array}{l}x_{g} \\ x_{p}\end{array}\right]=\left[\begin{array}{l}0 \\ 0\end{array}\right]$

The natural frequencies and the mode shapes $\left(\phi^{j}\right)$ of the system can be obtained by solving the eigenvalue problem normalized to $\phi^{T} M \phi=I$. For this case, the solution for the two-mode shapes is known [31] and corresponds to the coupled behavior.

When modal superposition is applied (Eq. (4)), the contribution of each mode $\left(\xi_{j}\right)$, including the damping effect of the coupled system $\left(\zeta_{j}\right.$ structure-impact element) [26], can be obtained through Eq. (5).

$\left[\begin{array}{l}x_{g} \\ x_{p}\end{array}\right]=\sum_{j=1}^{2} \xi_{j}(t) \cdot\left[\begin{array}{l}\phi_{g}^{j} \\ \phi_{p}^{j}\end{array}\right]$

$m_{j} \ddot{\xi}_{j}(t)+2 \zeta_{j} \omega_{n j} \dot{\xi}_{j}(t)+k_{j} \xi_{j}(t)=0$

A transient analysis with an initial velocity of the impact element is performed by using the initial velocity for each mode defined in Eq. (6). The time solution for the mode is shown in Eq. (7).

$\left[\begin{array}{l}\dot{\xi}_{1}(0) \\ \dot{\xi}_{2}(0)\end{array}\right]=\phi^{-1}\left[\begin{array}{l}0 \\ v_{0}\end{array}\right]$

$\xi_{j}(t)=e^{-\zeta \omega_{n j} t}\left(\frac{\dot{\xi}_{0}}{\sqrt{1-\zeta_{j}^{2}} \omega_{n j}} \sin \sqrt{1-\zeta_{j}^{2}} \omega_{n j} t\right)$

An acceleration time history of the pendulum is obtained from the second derivation of $x_{p}$ (Eq. (4)). The solution is calculated only during the contact stage (until the change of sign of the displacement).

With this solution, the parameters of the impact (maximum force, duration and impulse) can be easily calculated. Applied force is computed by multiplying the acceleration response by the impact element mass (Eq. (8)). The duration is defined by the elapsed time between the initial instant and the first time that the force value is zero after reaching its maximum value. Impulse is the momentum variation and is obtained with the integral of the force time history (Eq. (9)).

$F_{p}(t)=m_{p} \cdot \ddot{x}_{p}(t)$

$I_{p}=\Delta p=\int_{0}^{t} F_{p}(t) \cdot d t$

In the proposed mathematical model (Fig. 2), the degree of freedom of the pendulum is defined by its mass, according to the standard [25] $\left(m_{p}=50 \mathrm{~kg}\right.$ ), and its characteristic stiffness $k_{p}=350 \mathrm{kN} / \mathrm{m}$. For the glass plate, data from modal test developed on the test campaign are used.

\subsection{Tüme histories}

The German Standard DIN 18008 [13] includes in its appendix on the dynamic phenomenon during contact, the time histories of the acceleration of the pendulum head against a rigid wall and against a tempered eight $\mathrm{mm}$ thick glass measuring $1936 \times 876 \mathrm{~mm}$. The standard indicates that the latter comes from an experimental test. To check the validity of the 2DOF model, a comparison between the acceleration histories, provided by the German standard and the results obtained from the mathematical model, is performed.

The standard presents two impact energies (potential energy) with

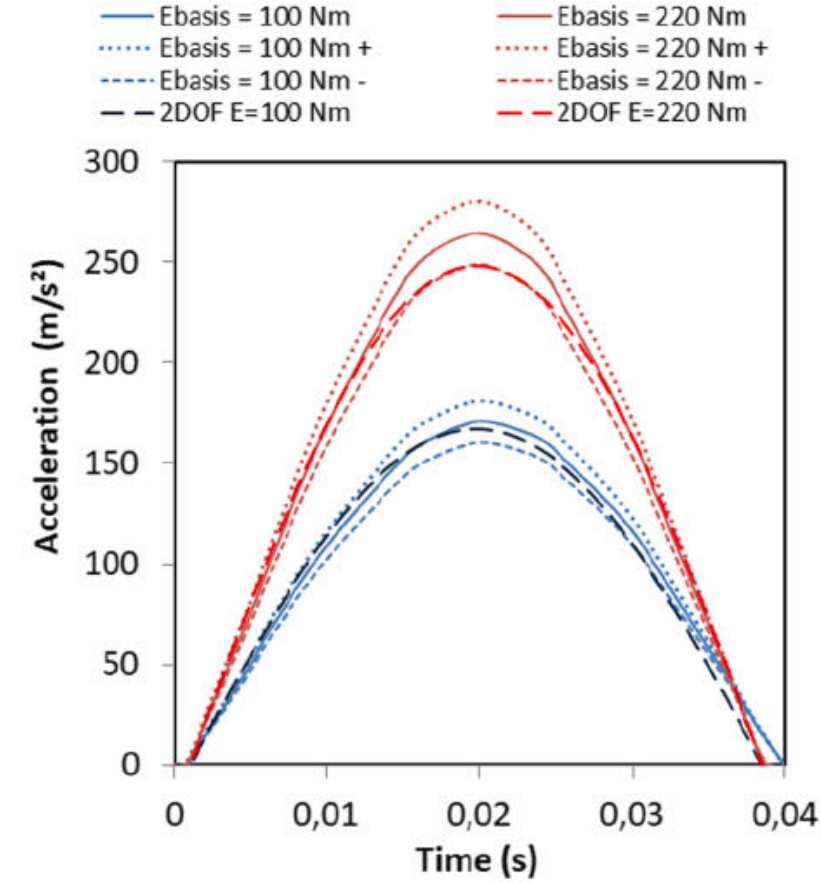

Fig. 3. Acceleration time history of the pendulum during impact on a rigid wall.

nominal values: Ebasis $=100 \mathrm{~J}$ and Ebasis $=220 \mathrm{~J}$. Each nominal curve is accompanied by two curves that set lower and higher permissible limits for impact similarity. In this case, a 2DOF solution is obtained, considering the stiffness of infinite glass and $350 \mathrm{kN} / \mathrm{m}$ for the impact element. Fig. 3 shows the acceleration time history of a pendulum impact against a rigid wall.

For low energy impacts, the maximum value obtained from the 2DOF is similar to the nominal, but the duration of the curve proposed by the standard is longer. This fact indicates an increase in the impact impulse. For the high energy impact, there are no significant differences in duration between standard and calculated impacts, but the maximum acceleration value is close to the lower limit curve. It seems that the standard uses a variable impact element stiffness (in most cases, unknown by the designer) in function with the impact level. Differences arise even in the simplest case.

If a real impact on a tempered eight mm thick glass is compared to a simplified model, there are greater differences between curves (Fig. 4). As in the case of a rigid wall, the duration at a high energy level is equal, but some variations appear for low energy levels. In this case, the shapes of the curves are slightly different. A higher contribution of the coupled second mode appears in the analytical simulation, thereby producing greater differences. It should be noted that the curves obtained from the simplified model for both energy levels can be considered a good approximation with similar maximum values. However, the assessment of the approximation level is difficult even including uncertainty curves. For soft impacts in flexible structures, the contribution of the second coupled mode (pendulum-glass) cannot be ignored. Thus, it is difficult to compare impacts from different sources. Clearly, there is a need for a procedure focused on the specific values to be evaluated, instead of a procedure which takes into account general aspects.

In light of the previous analysis, the most important values for comparing different curves are the duration (DP), maximum value (AP) and area under the curve (IP). These parameters can be obtained for any test if the acceleration of the impact head is recorded.

Each of these parameters presents relevant information about impact. The impulse (IP) describes the variation of momentum during an impact (energy change). The maximum force (AP) indicates the peak 


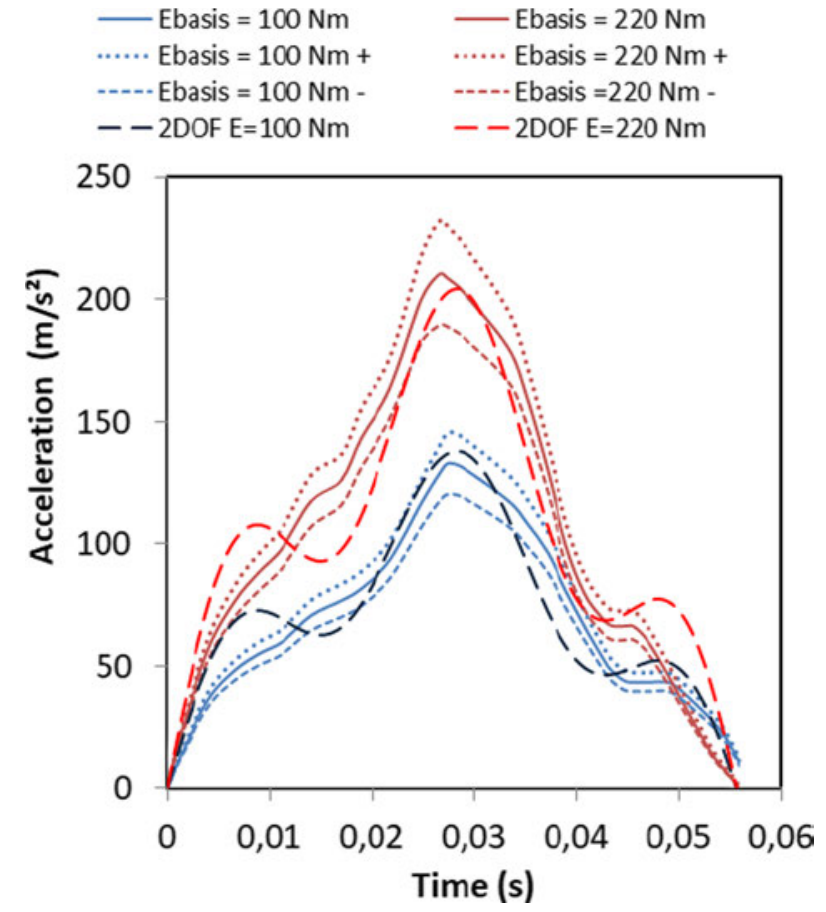

Fig. 4. Acceleration time history of the pendulum during impact on T08-4S.

value of the maximum transmitted force, and the duration (DP) is related to the stiffness of the elements involved in the response.

\section{Analysis of impact time histories}

\subsection{Test campaign description}

In order to develop a procedure for impact comparison, it is necessary to establish how to calculate the differences and to quantify these differences. For this purpose, it is essential to define an impact reference. This work has been carried out with the results obtained from an impact test campaign for glass according to the normative UNE12600 [25]. The data comes from a total of 21 glass plates divided into 7 different specimens (a total of 240 impacts). The size of the glass depends on the boundary conditions, testing glass with a size of $876 \times 1938 \mathrm{~mm}$ and supported on four sides (4S) and on 4 points (4P), and with a size of $876 \times 1200 \mathrm{~mm}$ and supported on two sides (2S). Regarding the thickness of the glass tested, 5 and $10 \mathrm{~mm}$ tempered glass plates were tested. In the case of laminated glass, it was produced with layered glass with thicknesses of $3+3 \mathrm{~mm}, 4+4 \mathrm{~mm}$ and $5+5 \mathrm{~mm}$. The interlayer material is comprised of polyvinyl butyral (PVB) and the total thickness of the glass is $6.39 \mathrm{~mm}(\mathrm{~L} 3+3), 8.01 \mathrm{~mm}(\mathrm{~L} 4+4)$ and $10.03 \mathrm{~mm}(\mathrm{~L} 5+5)$. All specimens were tested at different energy levels from $12 \mathrm{~J}$ to $590 \mathrm{~J}$.

This wide range of impact energies provides an overview of the impact behavior at different impact levels. The upper and lower limits are beyond the values that are usually considered characteristic in the study of soft impacts on glass in buildings. Some studies show that impact energies in soft human impact are much lower [32,33], but in characterizing the procedure, all the data will be used.

There is also a wide range of differences insofar as the thickness and boundary conditions of the glass used in the study. This allows us to study the differences between impacts with different structural behavior.

For the test campaign [24], a total of seven sensors were used. Fig. 5 shows the position of every sensor, four accelerometers, two strain gauges and a LVDT to record the height of the element impact. It should be noted that all these sensors adhere to the exhaustive analysis and

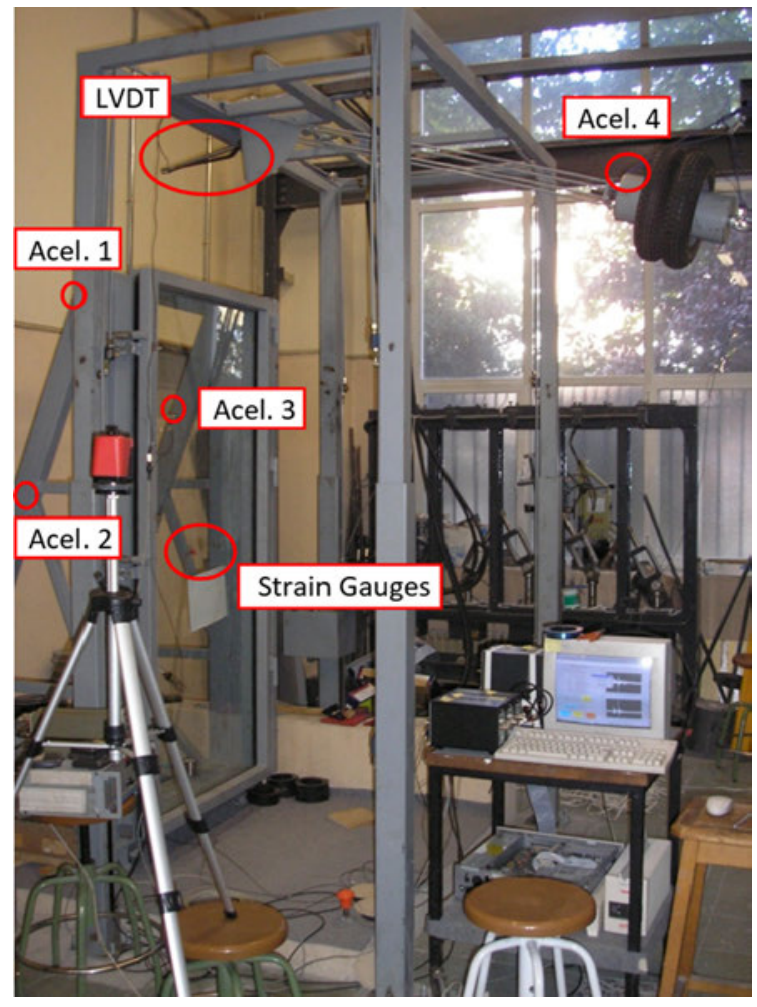

Fig. 5. Description of the positions of sensors in the pendulum test.

control in laboratory tests for a very detailed study of the impact phenomenon for research purposes. However, only accelerometer number four (see Fig. 5), located on the impact head, would be necessary to perform a comparison analysis of similarity between impacts.

To characterize the glass plates, modal tests on operational conditions were performed. Table 1 shows the frequency and modal point stiffness for each specimen. Modal point stiffness was obtained from the frequency response function (FRF) of the modal test. The number of impacts carried out for each type of glass is also presented. All impacts and modal test were carried out at room temperature. The name of the specimens is simplified with either $\mathrm{T}$ for tempered or $\mathrm{L}$ for annealed laminates, followed by the thickness of the layer/layers of glass and finally the boundary conditions (supported on 4S: four sides, 2S: two sides and at 4P: 4 points).

\subsection{Analysis of the parameters}

The value of the parameters have been obtained for all the impacts. In the case of the maximum acceleration (AP) and the impulse (IP), the analysis of the test results [24] shows the linear behavior of each parameter versus the nominal initial velocity of the impact head for each plate configuration. Therefore, a nominal reference impact (NRI) can be defined to estimate the impulse and the maximum acceleration,

Table 1

Description of the impact test campaign.

\begin{tabular}{lllll}
\hline Specimens & $\mathrm{N}^{\circ}$ impacts & $\begin{array}{l}\text { Tested energy } \\
\text { levels }\end{array}$ & $\begin{array}{l}\text { Modal stiffness } \\
(\mathrm{kN} / \mathrm{m})\end{array}$ & Frequency $(\mathrm{Hz})$ \\
\hline $\mathrm{T} 10-4 \mathrm{~S}$ & 54 & 9 & 807 & 35.22 \\
$\mathrm{~T} 05-4 \mathrm{~S}$ & 41 & 15 & 79 & 20.24 \\
$\mathrm{~T} 10-2 \mathrm{~S}$ & 12 & 4 & 573 & 31.58 \\
$\mathrm{~T} 10-4 \mathrm{P}$ & 28 & 7 & 58 & 8.70 \\
L55-4S & 51 & 14 & 881 & 36.04 \\
L44-4S & 17 & 8 & 342 & 30.48 \\
L33-4S & 37 & 13 & 157 & 25.03 \\
\hline
\end{tabular}



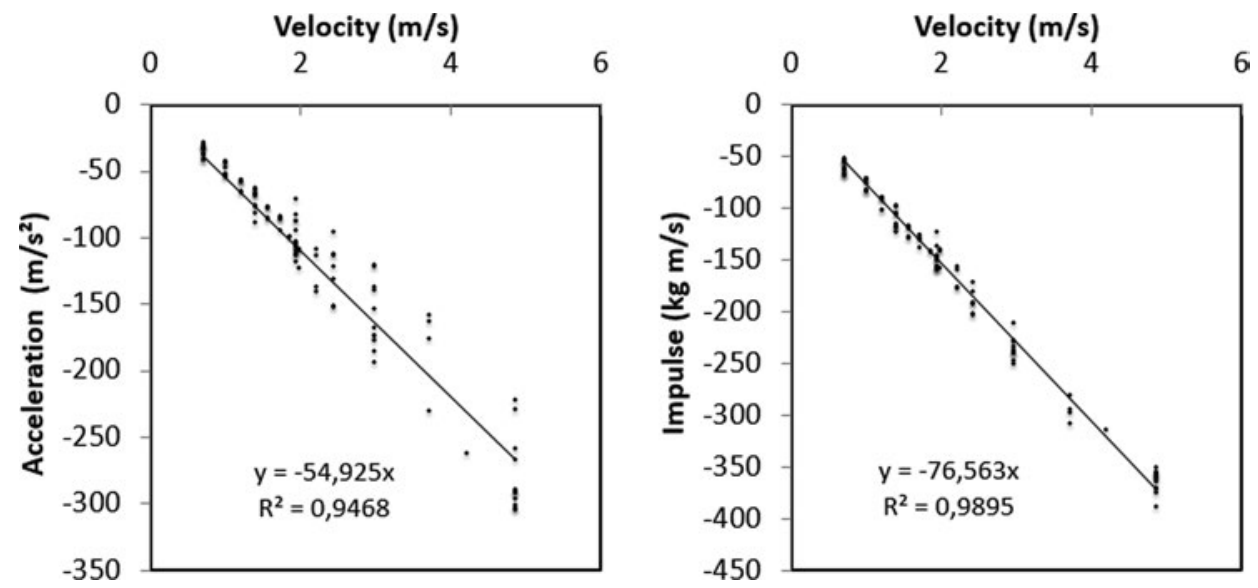

Fig. 6. Maximum acceleration (AP) and impulse (IP) recorded for all the recorded impacts during the test campaign.

as a function of the initial velocity. The values (AP and IP) obtained with these lines are useful for performing comparisons between impacts.

Analyzing all the impacts versus the nominal initial velocity (energy level), the acceleration (AP) and the impulse (IP) shows a good linear behavior but dispersion among the different configurations (structure sizes and boundary conditions) appears.

Fig. 6 shows the values of the acceleration and impulse parameters (AP and IP) as a function of the initial velocity obtained from the test. Regression lines for the total population data are also presented, and they are used to define nominal impacts (NRI).

In order to cover all cases, two configurations for nominal reference impact (NRI) are considered depending on the assessment purpose -general comparison (NRI-G) and repeatability comparison (NRI-R).

For general comparison, the data used to define the reference impact (NRI-G) includes all impacts, taking into account glass with different thicknesses, boundary conditions and dimensions.

For a repeatability comparison, the data include the tests carried out on glass T10-4S, because this glass is the standard reference [25]. By limiting the data to those obtained from the same type of glass tested with identical boundary conditions, the reference impact (NRI-R) corresponds with only one structure.

It may be observed that the impulse (IP) has a high linear correlation coefficient with the initial velocity $(\approx 0.99)$ demonstrating the importance of this parameter in the description of the impact level. Therefore, impulse shows great agreement in all the cases regardless of the boundary conditions or the dimensions of the tested glass (dynamic behavior between 8 and $40 \mathrm{~Hz}$ ). The parameters are defined within a range from 0 to $4.85 \mathrm{~m} / \mathrm{s}(\approx 590 \mathrm{~J})$ due to the broad range of the test campaign.

In the case of the maximum acceleration (AP), the regression coefficient is near 0.95 and the linear approach can be used for the NRI definition. Table 2 presents all the expressions needed to define the parameters (IP-AP) of the nominal reference impacts for the two comparison types (global-NRI-G and repeatability NRI-R). In addition, the correlation coefficient $\mathrm{R}^{2}$ for each parameter is shown.

The duration value obtained for each impact is more dependent on

Table 2

Nominal parameter definition: Regression analysis of impact test campaign. Values for AP and IP.

\begin{tabular}{llll}
\hline Reference parameter & & NRI-G & NRI-R \\
\hline$A P\left(\mathrm{~m} / \mathrm{s}^{2}\right)=\alpha v_{\text {pen }}(\mathrm{m} / \mathrm{s})$ & $\alpha$ & -54.925 & -62.970 \\
$I P \quad(\mathrm{kgm} / \mathrm{s})=\beta v_{\text {pen }}(\mathrm{m} / \mathrm{s})$ & $\beta$ & -76.563 & -79.375 \\
AP Correlation & $\mathrm{R}^{2}$ & 0.946 & 0.989 \\
$I P$ Correlation & $\mathrm{R}^{2}$ & 0.989 & 0.990 \\
\hline
\end{tabular}

Table 3

Nominal parameter definition: duration (DP) obtained with a 2DOF model.

\begin{tabular}{lll}
\hline Glass & 2DOF Mode $1(\mathrm{~Hz})$ & Duration $(\mathrm{s})$ \\
\hline T10-4S & 10.57 & 0.047 \\
T10-4P & 4.23 & 0.118 \\
T10-2S & 10.19 & 0.049 \\
T05-4S & 8.45 & 0.059 \\
L55-4S & 8.91 & 0.056 \\
L44-4S & 8.17 & 0.061 \\
L33-4S & 7.90 & 0.063 \\
\hline
\end{tabular}

the stiffness of the plate. For this reason, the mean value of the duration for each tested configuration is considered to complete the definition of the NRI. Table 3 shows the frequency of the first mode of coupled behavior of all tested glass obtained with the values of Table 1 and the 2DOF model. The duration corresponds with a half period.

\section{Comparison model: Similarity index}

\subsection{General description}

The proposed comparison model evaluates the approximation of the study impact (ImS) to the reference one (ImR), based on the relative differences of the characteristic parameters. Fig. 7 shows the impact force time history obtained multiplying the acceleration of the impact head by its mass. Considering T10-4S-E220 as the reference impact and T05-4S-E220 as the studied impact, one indicator (IPI, IPA and IPD) is

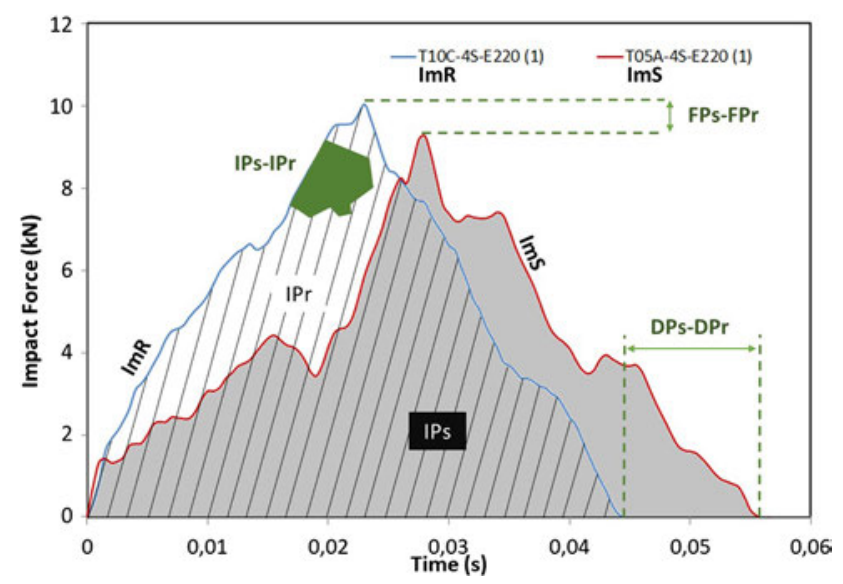

Fig. 7. Parameter differences between the Impact study (ImS) and reference Impact (ImR). 
defined for each parameter. Each of the three indicators may be defined by the equations, Eqs. (10), (11) and (12).

IPI $=\frac{\text { IPs }- \text { IPr }}{\text { IPr }}$

$\mathrm{IPA}=\frac{\mathrm{APs}-\mathrm{APr}}{\mathrm{APr}}=\frac{\mathrm{FPs}-\mathrm{FPr}}{\mathrm{FPr}}$

$\mathrm{IPD}=\frac{\mathrm{DPs}-\mathrm{DPr}}{\mathrm{DPr}}$

These indicators are normalized to obtain values between 0 and 1 $\left(I N D_{I M}, I N D_{F_{M A X}}\right.$ and $\left.I N D_{T}\right)$. Finally, the combination of them provides a global comparison value, which allows defining the similarity index (SIn) Eq. (13), for the study impact with respect to a reference one. Weighted coefficients $\left(\gamma_{i m}, \gamma_{f \max }\right.$ and $\left.\gamma_{t}\right)$ are defined for each indicator to calculate a similarity index. The similarity index (SIn) provides a value between 0 and 1 , where 1 signifies the highest degree of similarity and 0 the minimum. This value describes the global level of similarity between the study impact (ImS) and the reference impact (ImR). The SIn is defined as a weighted sum of the three normalized indicators: impulse, maximum force and duration.

$S I n=\gamma_{\text {im }} I N D_{I M}+\gamma_{\text {fmax }} I N D_{\text {FMAX }}+\gamma_{t} I N D_{T}$

To define the normalization of the indicators and their weighted coefficients, data from a human impact test campaign on glass according to the standard [25] are used. The glass plates were tested at different energy levels, that is, a total of 240 impacts on 7 different specimens, with impacts ranging from $12 \mathrm{~J}$ up to approximately $590 \mathrm{~J}$. The range of impacts considered in the study enable defining a sufficiently-large population of data to produce a comparison method.

\subsection{Normalization procedure}

The similarity indicators of each parameter are obtained based on the relative difference $\left(\delta_{i}\right)$ between the parameters of the study impact (IS) and the reference impact (IR) (Eq. (14)).

$\delta_{i}=\frac{I S_{i}-I R_{i}}{I R_{i}}$

A normalized value between 0 and 1 is assigned to each indicator by using a cosine function $\left(f\left(\left|\delta_{i}\right|\right)\right)$, which uses value 1 for identical impacts and 0 for the maximum difference allowed. Other functions, such as linear and exponential functions, were used, but only the cosine function provides satsifactory results for similar impacts with small differences. To establish the maximum relative difference $\left(\delta_{i}\right)$, a statistical analysis with the impacts of the test camping has been performed. The relative differences of each impact and the reference impact (NRI-G and NRI-R) are calculated for the total population. The standard deviations for each indicator and each type of analysis (NRI-G and NRI-R) are obtained.

For the normalization, a value of three times the standard deviation $\left(\sigma\left(\delta_{i}\right)\right)$ is used. Eq. (15) and Fig. 8 describe the used cosine function and the range in function of the standard deviation in which the indicator takes a value higher than 0 .

$I N D_{i}=f\left(\left|\delta_{i}\right|\right)\left\{\begin{aligned} 1 \leq f\left(\left|\delta_{i}\right|\right) \leq 0 & \text { if } 0 \leq\left|\delta_{i}\right| \leq \delta_{i}^{M A X} \\ f\left(\left|\delta_{i}\right|\right) & =0 \text { if }\left|\delta_{i}\right|>\delta_{i}^{M A X}\end{aligned}\right.$

\subsection{Standard deviations and weighted coefficients}

The first step is to obtain the standar desviation $\sigma\left(\delta_{i}\right)$ of the indicators (IPI, IPA and IPD) for the two cases: General comparison, and repeatability comparison. The data used for the statistical analysis are each one of the impact test, as study impact (ImS), and the Nominal Reference Impact (NRI), as reference impact (ImR). The second step is

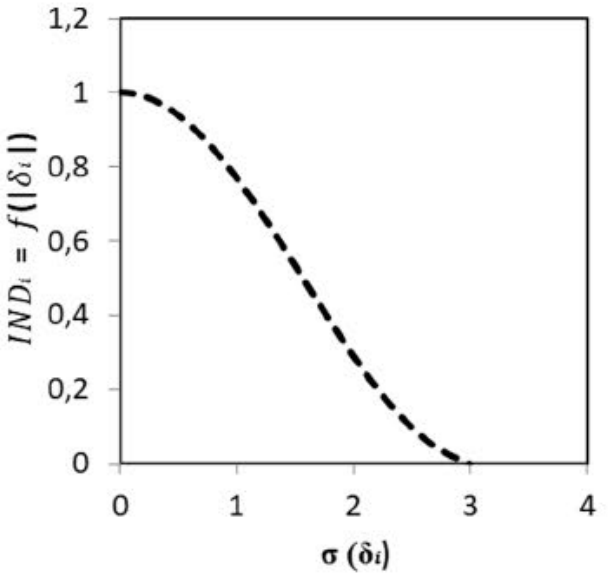

Fig. 8. Cosine function to assign the normalized indicator value.

the calculation of the normalized indicators (IND) using $3 \sigma\left(\delta_{i}\right)$.

To obtain the values of the weighted coefficients $\gamma_{i}$ an optimization procedure has been performed for each comparison purpose.

- Design parameters are the weighted coefficients $\gamma_{i}$ defined between 0 and 1 . Furthermore, their sum is equal to 1 .

- The Objetive function (Eq. (16)) is minimized. The SIn of each impact test with respect to that calculated with the nominal reference impact (NRI-G or NRI-R) are obtained ( $j=1$ to the maximum impact number). The $\operatorname{SIn}$ values should be near 1 and they are used to obtain the weighted coefficient.

$\min \sum_{j=1}^{t}\left(1-\operatorname{SIn} n^{2}\right.$

The application of the entire process described by the data of the glass impact test campaign may be summarized by the weighted coefficients and the standard deviation. Table 4 shows all the information needed to carry out a comparison of any impact for purposes of general comparison (CCG) and repeatability (CCR).

As an example, indicators of the procedure are applied to the impacts of the German standard for $220 \mathrm{~J}$, thereby considering the curve proposed by the standard as a reference impact (ImR) and the limits as a study impact (ImS). Table 5 sets forth the value of the three proposed parameters for comparison.

Table 6 presents the values for the three normalized indicators and the Similarity Index for the general and repeatability comparisons, and compares the nominal standard reference glass with the limit curves of the German standard.

\section{Applicability of the comparison procedure}

To assess the developed procedure, several comparisons are presented below using impacts from the test campaign, the German standard (GS) [13] and the mathematical results obtained from the 2DOF model. In the following examples, impacts from 100 and $220 \mathrm{~J}$ of energy basis are compared. The SIn is calculated in each case for both

Table 4

Similarity Index parameters - General and repeatability comparison.

\begin{tabular}{lcccccccc}
\hline \multirow{2}{*}{$\begin{array}{l}\text { Comparison } \\
\text { configuration }\end{array}$} & \multicolumn{3}{c}{ Weighted coefficients } & & \multicolumn{2}{c}{ Standard deviations } & \multirow{2}{*}{ (\%) } \\
\cline { 2 - 3 } & $\gamma_{i m}$ & $\gamma_{f \max }$ & $\gamma_{t}$ & & $\sigma_{i m}$ & $\sigma_{f \max }$ & $\sigma_{t}$ \\
\hline CCR & 0.392 & 0.508 & 0.1 & & 2.54 & 1.95 & 2.5 \\
CCG & 0.385 & 0.224 & 0.391 & & 7.78 & 12.35 & 8.80 \\
\hline
\end{tabular}


Table 5

Comparison parameters of the standard rigid wall impact GS-RIG-E220.

\begin{tabular}{llll}
\hline Impact & FP $(\mathrm{kN})$ & IP $(\mathrm{kg} \mathrm{m} / \mathrm{s})$ & DP $(\mathrm{ms})$ \\
\hline GS-RIG-E220 NOM & 13.23 & 302.87 & 38 \\
GS-RIG-E220 + & 14.02 & 321.04 & 38 \\
GS-RIG-E220 - & 12.44 & 284.69 & 38 \\
\hline
\end{tabular}

Table 6

Comparison results of the standard rigid wall impact GS-RIG-E220.

\begin{tabular}{llllll}
\hline Impact & Comparison & SIn & IND $_{\mathrm{F}_{\mathrm{MAX}}}$ & IND $_{\mathrm{IM}}$ & IND $_{\mathrm{T}}$ \\
\hline \multirow{2}{*}{ GS-RIG-E220 + } & CCG & 0.93 & 0.94 & 0.85 & 1 \\
& CCR & 0.14 & 0 & 0.11 & 1 \\
GS-RIG-E220 - & CCG & 0.93 & 0.94 & 0.85 & 1 \\
& CCR & 0.14 & 0 & 0.11 & 1 \\
\hline
\end{tabular}

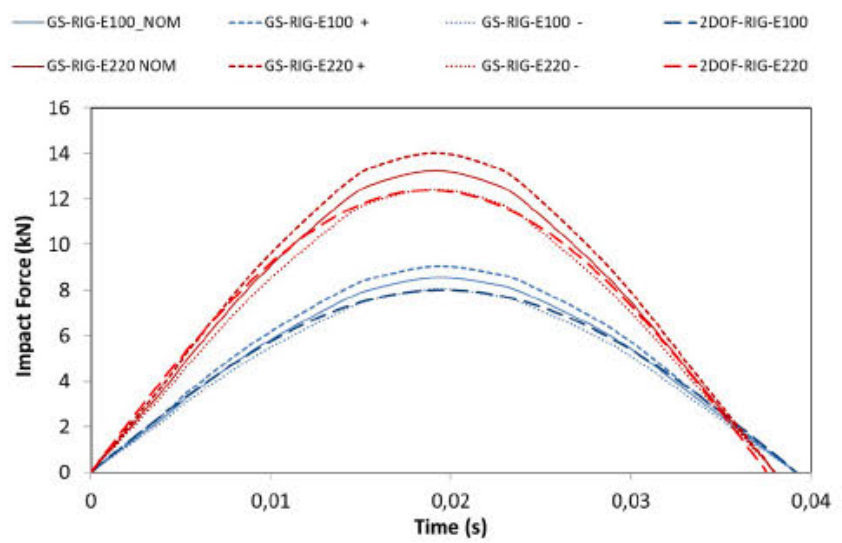

Fig. 9. Rigid Wall Comparison; GS \& 2DOF - Time history.

repeatability and general configurations.

\subsection{Evaluation of the similarity between the permissible standard limits}

The first step is to study the level of similarity for the limits provided by the German standard for a rigid wall [13]. The nominal impact from the standard is the reference impact (ImR) and the upper and lower limits and the impact calculated with the 2DOF model are the studied impacts (ImS) (Fig. 9). Table 7 presents the similarity index and the value of all indicators for both comparison methods, that is the general comparison and repeteability comparison.

For the standard impacts, the Similarity Index (Table 7) for nominal cases versus maximum and minimum limits are the same: 0.93 for the general comparison and 0.14 for the repeatability comparison.

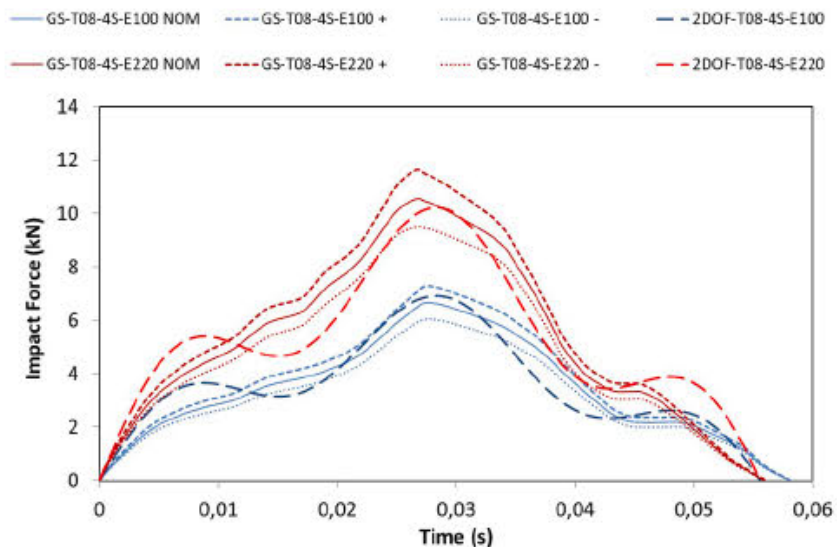

Fig. 10. T08-4S - Impact Comparison - Time history.

Therefore, the acceleration interval provided by the standard can be measured by the Similarity Index procedure.

In the case of the upper and lower curves, the force values and the duration indicator for both impact levels are similar in general and repeatability comparisons. Differences in SIn arise from the Impulse indicator INDimp (0.85-0.11), thereby showing the importance of this variable.

The numerical differences of the indicators, using the global and repeatability model provide the possibility of performing a more detailed evaluation. If general comparison indicators are high, a repeatability analysis provides additional information about the differences between impacts.

The comparison of the 2DOF model (ImS) with the nominal impact of the standard (ImR), produces different results for the two energy levels with higher SIn and indicators than the previous ones. Therefore, the differences of the model results are within the range accepted by the standard.

For the second step, the comparisons involve the impacts provided by the standard on a tempered glass with a thickness of $8 \mathrm{~mm}$ (Fig. 10). As in the previous case, the nominal curve is considered a reference impact (ImR) and compared to the upper and lower limits of the standard and a 2DOF calculation (ImS). Even though the simplified approach is outside of the standard limit curves in some time intervals, applying the SIn reveals that the 2DOF approach can be considered better than the limit impacts provided by the standard (Table 8 ). The SIn behavior in this comparison is very similar to the previous case.

The values of the SIn obtained from the standard impacts can be used as a reference to define permissable values that can be used to accept any impact related to the standard.

Repeatability values can be used to compare two equal impact tests to check the acceptability of the test itself. To check the approach of an

Table 7

Rigid wall comparison; GS \& 2DOF - results.

\begin{tabular}{|c|c|c|c|c|c|c|c|c|}
\hline \multirow[t]{2}{*}{ GLASS TYPE } & \multicolumn{2}{|c|}{ Sin } & \multicolumn{2}{|c|}{ IND $_{\text {FMAX }}$} & \multicolumn{2}{|c|}{$\mathrm{IND}_{\mathrm{IM}}$} & \multicolumn{2}{|c|}{$\mathrm{IND}_{\mathrm{T}}$} \\
\hline & CCG & CCR & CCG & CCR & CCG & CCR & CCG & CCR \\
\hline \multicolumn{9}{|c|}{ IMPACT ENERGY $=100 \mathrm{~J}$ - REFERENCE IMPACT: GS-RIG-E100 NOM } \\
\hline GS-RIG-E100 + & 0.93 & 0.14 & 0.94 & 0.00 & 0.85 & 0.11 & 1.00 & 1.00 \\
\hline GS-RIG-E100 - & 0.93 & 0.14 & 0.94 & 0.00 & 0.85 & 0.11 & 1.00 & 1.00 \\
\hline 2DOF-RIG-E100 & 0.98 & 0.45 & 0.93 & 0.00 & 0.99 & 0.89 & 1.00 & 1.00 \\
\hline \multicolumn{9}{|c|}{ IMPACT ENERGY $=220 \mathrm{~J}$ - REFERENCE IMPACT: GS-RIG-E220 NOM } \\
\hline GS-RIG-E220 + & 0.93 & 0.14 & 0.94 & 0.00 & 0.85 & 0.11 & 1.00 & 1.00 \\
\hline GS-RIG-E220 - & 0.93 & 0.14 & 0.94 & 0.00 & 0.85 & 0.11 & 1.00 & 1.00 \\
\hline 2DOF-RIG-E220 & 0.97 & 0.40 & 0.93 & 0.00 & 0.98 & 0.80 & 0.99 & 0.93 \\
\hline
\end{tabular}


Table 8

T08-4S - impact comparison - results.

\begin{tabular}{|c|c|c|c|c|c|c|c|c|}
\hline \multirow[t]{2}{*}{ GLASS TYPE } & \multicolumn{2}{|c|}{ Sin } & \multicolumn{2}{|c|}{ IND $_{\text {PMAX }}$} & \multicolumn{2}{|c|}{$\mathrm{IND}_{\mathrm{IM}}$} & \multicolumn{2}{|c|}{$\mathrm{IND}_{\mathrm{T}}$} \\
\hline & CCG & CCR & CCG & CCR & CCG & CCR & CCG & CCR \\
\hline \multicolumn{9}{|c|}{ IMPACT ENERGY $=100 \mathrm{~J}$ - REFERENCE IMPACT: GS-T08-4S-E100 NOM } \\
\hline GS-T08-4S-E100 + & 0.85 & 0.10 & 0.85 & 0.00 & 0.70 & 0.00 & 1.00 & 1.00 \\
\hline GS-T08-4S-E100 - & 0.85 & 0.10 & 0.85 & 0.00 & 0.70 & 0.00 & 1.00 & 1.00 \\
\hline 2DOF-T08-4S-E100 & 0.97 & 0.57 & 0.98 & 0.29 & 1.00 & 0.98 & 0.94 & 0.40 \\
\hline \multicolumn{9}{|c|}{ IMPACT ENERGY $=220 \mathrm{~J}$ - REFERENCE IMPACT: GS-T08-4S-E220 NOM } \\
\hline GS-T08-4S-E220 + & 0.85 & 0.10 & 0.83 & 0.00 & 0.71 & 0.00 & 1.00 & 1.00 \\
\hline GS-T08-4S-E220 - & 0.85 & 0.10 & 0.83 & 0.00 & 0.71 & 0.00 & 1.00 & 1.00 \\
\hline 2DOF-T08-4S-E220 & 0.99 & 0.74 & 0.99 & 0.52 & 1.00 & 0.96 & 1.00 & 0.99 \\
\hline
\end{tabular}

$\begin{array}{cccc}\text {-T10C-4S-E100 (1) } & \cdots-\text { T10C-4S-E100(2) } & \text { T10C-4S-E95 (1) } & - \text { T10C-4S-E95 (2) } \\ \text {-TOSA-4S-E220 (1) } & \cdots-\text { TOSA-4S-E220(2) } & \cdots-\text { TOSB-4S-E220 (1) }\end{array}$

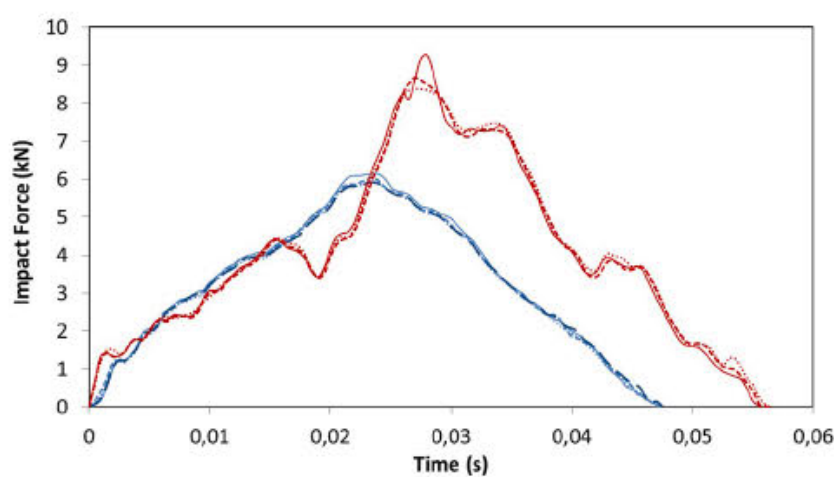

Fig. 11. Repeatability T10-4S and T05-4S - Impact Comparison - Time history.
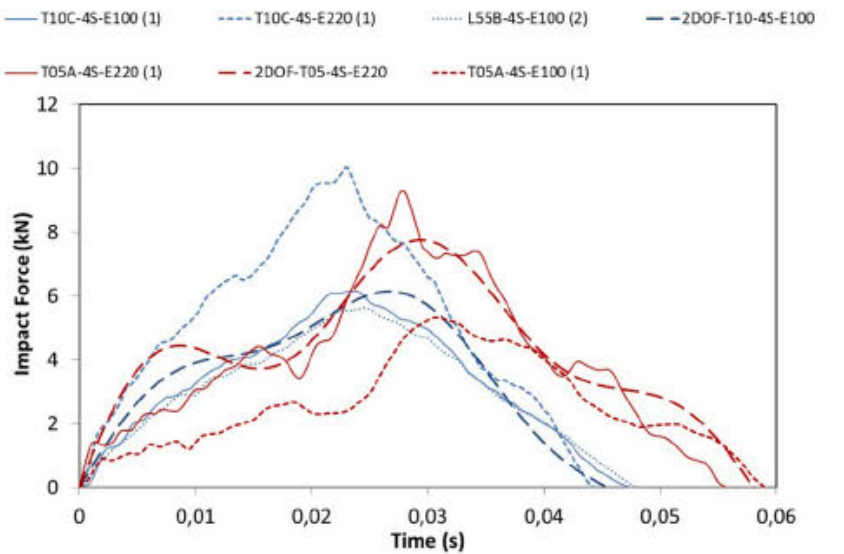

Fig. 12. T10-4S \& T05-4S -Impact Comparison - Time history.

Table 9

Repeatability T10-4S and T05-4S - impact comparison - results.

\begin{tabular}{|c|c|c|c|c|c|c|c|c|}
\hline \multirow[t]{2}{*}{ GLASS TYPE } & \multicolumn{2}{|c|}{ Sin } & \multicolumn{2}{|c|}{ IND $_{\text {PMAX }}$} & \multicolumn{2}{|c|}{$\mathrm{IND}_{\mathrm{IM}}$} & \multicolumn{2}{|c|}{$\mathrm{IND}_{\mathrm{T}}$} \\
\hline & CCG & CCR & CCG & CCR & CCG & CCR & CCG & CCR \\
\hline \multicolumn{9}{|c|}{ IMPACT ENERGY $=100 \mathrm{~J}-$ REFERENCE IMPACT: T10C-4S-E100 (1) } \\
\hline T10C-4S-E100 (2) & 1.00 & 0.86 & 0.99 & 0.76 & 0.99 & 0.94 & 1.00 & 1.00 \\
\hline T10C-4S-E95 (1) & 0.98 & 0.59 & 0.98 & 0.39 & 0.97 & 0.73 & 1.00 & 1.00 \\
\hline T10C-4S-E95 (2) & 0.99 & 0.58 & 0.97 & 0.28 & 0.99 & 0.87 & 1.00 & 1.00 \\
\hline \multicolumn{9}{|c|}{ IMPACT ENERGY $=220 \mathrm{~J}-$ REFERENCE IMPACT: T05A-4S-E220 (1) } \\
\hline T05A-4S-E220 (2) & 0.98 & 0.49 & 0.93 & 0.00 & 1.00 & 0.99 & 1.00 & 1.00 \\
\hline T05B-4S-E220 (1) & 0.96 & 0.47 & 0.85 & 0.00 & 0.99 & 0.95 & 1.00 & 1.00 \\
\hline
\end{tabular}

impact test with the standard reference, a general comparison configuration can be used.

\subsection{Evaluation of test repeatability}

In order to verify the repeatability of the phenomenon and the test set up in the test campaign, each impact (glass plate - initial energy) was recordered three times (referred by a number between parentheses). In addition, three different plates of each specimen were tested. In order to identify the tested plate, a letter was incorporated after the glass thickness (A, B, C). The curves are quite similar (Fig. 11), but in order to quantify differences, the SIn have been calculated.

Various examples are collected in Table 9: tempered glass with a thickness of $10 \mathrm{~mm}$ and an energy of $100 \mathrm{~J}$ and two additional cases with $5 \%$ less energy. For $220 \mathrm{~J}$, a tempered glass with a thickness of $5 \mathrm{~mm}$ is compared.

A general comparison indicates values close to one in all the indicators (Table 9). The evaluation, using the repeatability configuration of the method, permits studying the differences. Focusing on the final
SIn, the method is able to better detect similarity of the impacts from the same energy level. Indicators of the comparisons of impacts with small differences in initial energy are high, but lower than those obtained for impacts with the same initial energy. The indicators for the maximum forces are clearly lower than the impulse ones. The differences of the peak values can be observed on the plots, but seem to be insignificant while the values of the indicators are high. The actual importance of the differences between the maximum is not very high, as is shown by the SIn that reflects the great agreement of the curves.

\subsection{Evaluation of model results}

To assess the SIn model with impacts of different characteristics, the results of different plates are compared (T-10-4S, L55-4S and T05-4S) in Fig. 12. In addition, the transient response of the 2DOF model is included. Different comparisons between described impacts are presented in Table 10.

In almost all cases, the SIn obtained from the repeatability model is very low and no information is gained from analyzing these values. A 
Table 10

T10-4S \& T05-4S - impact comparison - results.

\begin{tabular}{|c|c|c|c|c|c|c|c|c|}
\hline \multirow[t]{2}{*}{ GLASS TYPE } & \multicolumn{2}{|c|}{ Sin } & \multicolumn{2}{|c|}{ IND $_{\text {FMAX }}$} & \multicolumn{2}{|c|}{$\mathrm{IND}_{\mathrm{IM}}$} & \multicolumn{2}{|c|}{$\mathrm{IND}_{\mathrm{T}}$} \\
\hline & CCG & CCR & CCG & CCR & CCG & CCR & CCG & CCR \\
\hline \multicolumn{9}{|c|}{ IMPACT ENERGY = $100 \mathrm{~J}$ - REFERENCE IMPACT: T10C-4S-E100 (1) } \\
\hline 2DOF-T10-4S-E100 & 0.94 & 0.68 & 1.00 & 0.98 & 0.90 & 0.32 & 0.94 & 0.45 \\
\hline L55B-4S-E100 (2) & 0.93 & 0.19 & 0.88 & 0.00 & 0.89 & 0.24 & 1.00 & 0.95 \\
\hline T05A-4S-E100 (1) & 0.51 & 0.16 & 0.71 & 0.00 & 0.92 & 0.41 & 0.00 & 0.00 \\
\hline \multicolumn{9}{|c|}{ IMPACT ENERGY $=220 \mathrm{~J}-$ REFERENCE IMPACT: T05A-4S-E220mm (1) } \\
\hline 2DOF-T05-4S-E220 & 0.79 & 0.07 & 0.59 & 0.00 & 0.71 & 0.00 & 0.97 & 0.65 \\
\hline T05A-4S-E100 (1) & 0.35 & 0.02 & 0.00 & 0.00 & 0.00 & 0.00 & 0.90 & 0.20 \\
\hline T10C-4S-E220 (1) & 0.59 & 0.18 & 0.88 & 0.00 & 0.93 & 0.47 & 0.09 & 0.00 \\
\hline
\end{tabular}

SIn value of 0.68 is reached only for the 2DOF model and test comparisons. This value increases to 0.94 for the general comparison model, so only the general comparison model is considered in the following paragraphs.

By comparing a 2DOF model with test results, great agreement is obtained for the T10C-4S-E100 test with a SIn value of 0.94 and three indicators higher than 0.9. In the case of the T05A-4S-E220 test, a lower SIn is obtained (0.79) due to the difficulty of representing the nonlinear behavior of the plate.

When two plates with similar stiffness are tested with same impact level, (T10-4S and L55-4S), a high SIn is obtained (0.93), but impulse and duration indicators are lower than that obtained for impacts on the same glass. If the stiffness of the glass is clearly different (T10-4S and T05-4S), the SIn is lower ( 0.51 at $100 \mathrm{~J}$ and 0.59 at $220 \mathrm{~J})$. The energy of both impacts is similar, that is they have a high impulse indicator (0.92-0.93). However, the duration indicator shows that the structures are clearly different (0-0.09).

Finally, if two impacts with different impact levels for the same glass are compared (T05-4S at $100 \mathrm{~J}$ and $220 \mathrm{~J}$ ), the SIn indicator is low and has low impulse and force indicators $(0-0)$, but with a higher duration indicator (0.90).

The previous results demonstrate that the energy level of two impacts can be compared to the impulse indicator. Likewise, the duration indicator is the best parameter for analyzing the similarity between the impacted structures.

\section{Conclusions}

This paper proposes a method for comparing transient impact histories by considering a comprehensive analysis of all the relevant parameters involved. Based on the knowledge acquired in the study of soft impacts on glass, a procedure has been developed to evaluate the level of similarity between impacts. The proposed procedure is applicable to transient time histories obtained from any type of source: tests, simplified models, standards or even finite element models.

The comparison method provides a similarity index value (SIn) for the global evaluation of the transient registers and three normalized indicators for detecting the source of the differences between impacts. This feature converts the proposed comparative process into a comprehensive tool, which goes beyond a mathematical comparison of functions. It allows evaluating the physical behavior of the impact from its most important parameters: maximum force, impulse and duration. All of them can be easily calculated from a time history of the acceleration of the impact head obtained from models or with an accelerometer located on the impacting element.

Using data from a test campaign of soft human impact against glass, two different comparison configurations have been set up: one to assess the repeatability of similar impacts and another one to evaluate generic impacts. Both configurations have been verified with different examples, thereby combining data from simplified 2DOF models, tests and normatives.
Examples show that the similarity index and the indicators give accurate information on the similarity between impacts, indicating the parameter that produces the differences between results.

The impact range provided by the German standard shows a SIn of 0.10 for repeatability purposes, while the analysis of the repeatability of test results compared in this paper show SIn values higher than 0.95. Therefore, it seems that the standard defines permissable impacts with a general comparison index between 0.85 and 0.93 . According to this range, the predictions of a 2DOF model compared to the tested glass plates (T10 and T05), may be acceptable as a valid approach to the impact with SIn of 0.79 and 0.94 .

The proposed comparison tool can be used to perform parametric studies that are simplified in order to select a better approach: only four numbers with physical meaning are necessary instead of a time history.

The set-up of the comparison tool has been carried out in this paper with data from soft impacts on glass. Standard deviation and coefficient values provided can be used for any type of impact with similar characteristics. If the comparison purpose or the type of impact is different, all the required steps to update the weighted coefficients and standard desviation are provided.

The study of new weighted coefficients and standard deviation of the indicators could be used for other applications, such a "in situ" testing, thereby helping to achieve even greater accuracy by expanding the current database with more impacts.

\section{References}

[1] Lam NTK, Gad EF, Nurhuda I, Calderone I. Impact resistance of annealed glass panels. J Perform Constr Facil 2011;25:422-32. https://doi.org/10.1061/ (ASCE)CF 1943-5509.0000181.

[2] Kursun A, Senel M. Investigation of the effect of low-velocity impact on composite plates with preloading. Exp Tech 2013;37:41-8. https://doi.org/10.1111/j.17471567.2011.00738.x.

[3] Schneider J, Schula S. Simulating soft body impact on glass structures. Proc Inst Civ Eng Build 2016;169:416-31. https://doi.org/10.1680/jstbu.13.00112.

[4] Yang Y, Lam NTK, Zhang L. Estimation of response of plate structure subject to low veloctiy impact by a solid object. Int J Struct Stab Dyn 2012;12. https://doi.org/10. 1142/S0219455412500538.

[5] Ledbetter SR, Walker AR, Keiller AP. Structural use of glass. J Archit Eng 2006;12:137-49. https://doi.org/10.1061/(ASCE)1076-0431(2006) 12:3(137).

[6] TRAV. Technische Regeln für die Verwendung von absturzsichernden Verglasungen. Deutsches Institut für Bautechnik (DIBt); 2003.

[7] Rück R, Weschler M. Numerical simulation of the pendulum test with a glass plate. Otto-Graf-J 2000;11:109-22.

[8] Schneider J. Festigkeit und Bemessung punktgelagerter Gläser und strossbeanspruchter Gläser. Doctoral thesis, Darmstadt. Technische Universität Darmstadt; 2001.

[9] Schneider J, Bohmann D. Glasscheiben unter Stoßbelastung - experimentelle und theoretische Untersuchungen für absturzsichernde Verglasungen bei weichem Stoß. Bauingenieur 2002;77:581-92.

[10] Brendler S. Rechnerisches Bemessungskonzept für absturzsichernde Glastafeln. Doctoral thesis, Institut für Bauwerkserhaltung und Tragwerk. TU Braunschweig; 2007.

[11] Schneider Jens, Schula Sebastian, Burmeister Albrecht. Zwei Verfahren zum rechnerischen Nachweis der dynamischen Beanspruchung von Verglasungen durch weichen Stoß: Teil 1: Numerische, transiente Simulationsberechnung und vereinfachtes Verfahren mit statischen Ersatzlasten. Stahlbau 2011;80(S1):81-7. https://doi.org/10.1002/stab.v80.3s10.1002/stab.201120010. 
[12] Weller Bernhard, Reich Stefan, Krampe Philipp. Zwei Verfahren zum rechnerischen Nachweis der dynamischen Beanspruchung von Verglasungen durch weichen Stoß: Teil 2: Numerische Vergleichsberechnungen und experimentelle Verifikation. Stahlbau 2011;80(S1):88-92. https://doi.org/10.1002/stab.v80.3s10.1002/stab. 201120011.

[13] DIN 18008-4: Glass in building - design and construction rules - Part 4: Additional requirements for barrier glazing. Berlin, Germany; 2013.

[14] Schneider J, Schula S. Transient impact simulation and a simplified mechanical model for soft body impact on glass panes. In: Int conf glas, Düsseldorf, Germany; 2010. p. 71-82.

[15] Schneider J, Wörner J-D. Impact loading on glass panes by soft body impact theoretical analysis and experimental verification. Glas Process Days 2001:682-7.

[16] Froling M, Persson K, Austrell P-E. A reduced model for the design of glass struc tures subjected to dynamic impulse load. Eng Struct 2014;80:53-60. https://doi. org/10.1016/j. engstruct.2014.08.043.

[17] Russell DM. Error measures for comparing transient data. In: 68th shock vib symp. Hunt Valley, Maryland: General Dynamics. Electric Boat; 1997.

[18] Wang Q, Gabler HC. Review of correlation methods for evaluating finite element simulations of impact injury risk. Biomed Sci Instrum 2008;44:268-73.

[19] Fam A, Rizkalla S. Laboratory simulation of impact loading on laminated glass for ice hockey arenas. Exp Tech 2007;31:46-52. https://doi.org/10.1111/j.1747-1567. 2007.00181.x.

[20] El-Dakhakhni WW, Mekky WF, Rezaei SHC. Validity of SDOF models for analyzing two-way reinforced concrete panels under blast loading. J Perform Constr Facil 2010;24:311-25. https://doi.org/10.1061/(ASCE)CF.1943-5509.0000090.

[21] Safari KH, Zamani J, Khalili SMR, Jalili S. Experimental, theoretical, and numerical studies on the response of square plates subjected to blast loading. J Strain Anal Eng Des 2011 ; 46. doi:10.1177/0309324711416183.

[22] Zhang X, Hao H, Wang Z. Experimental study of laminated glass window responses under impulsive and blast loading. Int J Impact Eng 2015;78:1-19. https://doi.org/ 10.1016/j.ijimpeng.2014.11.020.
[23] Oketami Y, Kikuta M, Aratani S. Investigation of repeatability and reproducibility of the shot bag impactor. Glas Process Days; 2001.

[24] Pacios A, Postigo S, Huerta C. Relationship between characteristic parameters of impact test for safety glasses. Stahlbau 2011:61-6. https://doi.org/10.1002/stab. 201120008.

[25] CEN. EN 12600: Glass in building - pendulum test - impact test method and classification for flat glass; 2002.

[26] Parra JA, Alonso J, Pacios A, Huerta MC. Effective energy applied to a glass plate during an impact test. Int J Impact Eng 2019;130:11-8. https://doi.org/10.1016/j. ijimpeng.2019.03.008.

[27] Chopra AK. Dynamic of structures-theory and applications to earthquake engineering. Upper Saddle River (NJ): Pearson Prentice Hall; 2007.

[28] Postigo S. Estudio teórico experimental de impactos humanos contra vidrios de acristalamientos de edificación (Doctoral thesis). Universidad Politécnica de Madrid; 2010.

[29] Sanchidrian A, Parra JA, Alonso J, Pecharroman J, Huerta C. Influence of differen boundary conditions of glass plates on human impact resistance. In: Cardiel VC, Perez MC, editors. 4TH int conf mech model struct eng (CMMOST 2017), CALLE RAMIRO MAEZTU 7, MADRID, 28040, SPAIN: UNIV POLITECNICA MADRID; 2017 p. $371-86$.

[30] Alonso J, Parra JA, Huerta MC. Simplified model for the behavior of human impact against glass plates. In: Cardiel VC, Perez MC, editors. 4TH int conf mech model struct eng (CMMOST 2017), CALLE RAMIRO MAEZTU 7, MADRID, 28040, SPAIN: UNIV POLITECNICA MADRID; 2017. p. 105-15.

[31] Blevins RD. Formulas for natural frequency and mode shape. Krieger Publishing Company; 2001.

[32] Seeger T. Skriptum Werkstoffmechanik/Werkstoffechnologie. TH-Darmstadt: Inst Für Stahlbau Und Werkstoffmechanik; 1995.

[33] Schneider J, Wörner J-D. Abschlussbericht zur experimentellen und rechnerischen Bestimmung der dynamischen Belastung von Verglasungen durch weichen Stoss. Forschungsbericht Für Das Dtsch Inst Für Bautechnik; 2000. 